\title{
Three-Wavelength $\mathrm{Tm}^{3+}$ :ZBLAN Fiber Laser and its Applications in Water Detection
}

\author{
Chenglai Jia ${ }^{1}$, Kishor Ramaswamy ${ }^{1}$, Lawrence Chen ${ }^{1}$, Amy G. MacLean ${ }^{2}$, Nicholas L.P. Andrews ${ }^{2}$, \\ John Saunders ${ }^{2}$, Jack A. Barnes ${ }^{2}$, Hans-Peter Loock ${ }^{2}$, and M. Saad ${ }^{3}$ \\ ${ }^{1}$ Department of Electrical and Computer Engineering, McGill University \\ Montreal, QC, H3A 0E9, Canada \\ ${ }^{2}$ Department of Chemistry, Queen's University, Kingston, ON, K7L 3N6, Canada \\ ${ }^{3}$ Thorlabs, Inc., 56 Sparta Ave., Newton, NJ 07860
}

\begin{abstract}
We demonstrate a three-wavelength $\mathrm{Tm}^{3+}$ :ZBLAN fiber laser emitting simultaneously at $1460 \mathrm{~nm}$, $1503 \mathrm{~nm}$, and $1873 \mathrm{~nm}$ and its application in single-pass absorption measurements for detecting and quantifying water concentration in acetone.
\end{abstract}

\section{INTRODUCTION}

Thulium-doped $\left(\mathrm{Tm}^{3+}\right)$ ZBLAN fiber provides a variety of energy transitions which can be used to develop fiber lasers operating at different wavelengths. Due to the lower phonon energy of ZBLAN glass compared with silica [1], the ${ }^{3} \mathrm{H}_{4} \rightarrow{ }^{3} \mathrm{~F}_{4}$ transition is capable of providing effective lasing around $1480 \mathrm{~nm}$. In addition, the lower attenuation around $2 \mu \mathrm{m}$ in ZBLAN glass makes it efficient for lasing around $1900 \mathrm{~nm}$ through the ${ }^{3} \mathrm{~F}_{4} \rightarrow{ }^{3} \mathrm{H}_{6}$ transition.

Multi-wavelength ZBLAN fiber lasers in the nearinfrared (NIR) range have potential applications in chemical detection and sensing [2]. By measuring absorption patterns at several wavelengths, they can be used to detect chemicals with improved sensitivity and specificity. We have previously demonstrated dualwavelength lasing around $1480 \mathrm{~nm}$ through the ${ }^{3} \mathrm{H}_{4} \rightarrow{ }^{3} \mathrm{~F}_{4}$ transition [3], as well as a dual-band fiber laser operating simultaneously at $810 \mathrm{~nm}$ and $1480 \mathrm{~nm}$ [4]. However, none of the previous reports made an attempt to apply the multiwavelength functionality for chemical detection or sensing.

In this paper, we demonstrate a three-wavelength $\mathrm{Tm}^{3+}$ :ZBLAN fiber laser emitting simultaneously at 1460 $\mathrm{nm}, 1503 \mathrm{~nm}$, and $1873 \mathrm{~nm}$. We investigate the laser output characteristics and present its application for water detection using single-pass absorption measurements.

\section{EXPERIMENTAL SETUP}

A schematic of the three-wavelength laser is shown in Fig. 1. It includes two independent cavities - the top branch to generate lasing at 1460/1503 $\mathrm{nm}$ and the bottom branch to generate lasing at $1873 \mathrm{~nm}$ - along with a third shared branch. In the top branch, up-conversion pumping from a $1064 \mathrm{~nm}$ ytterbium-doped fiber laser $\left(\mathrm{P}_{1064}, \mathrm{YDFL}\right)$ is used. The pump is coupled via a 1064/1480 nm wavelength division multiplexer (WDM) into a $52 \mathrm{~cm}$ length of $\mathrm{Tm}^{3+}$ :ZBLAN fiber. Two SMF-28 fiber Bragg gratings (FBGs) placed on one end serve as the wavelength selectors. FBG $\mathrm{FB}_{1}$ has a center wavelength $\lambda_{1}=1503 \mathrm{~nm}$, while $\mathrm{FBG}_{2}$ has a center wavelength $\lambda_{2}=1460 \mathrm{~nm}$. A polarization controller (PC) is placed between $\mathrm{FBG}_{1}$ and $\mathrm{FBG}_{2}$ in order to balance the powers generated at $\lambda_{1}$ and $\lambda_{2}$. In the bottom branch, a $1560 \mathrm{~nm}$ pump $\left(\mathrm{P}_{1560}\right)$ comprised of an external cavity laser (ECL) and an erbium-doped fiber amplifier (EDFA) is coupled through $\mathrm{FBG}_{3}$ into a $85 \mathrm{~cm}$ length of $\mathrm{Tm}^{3+}$ :ZBLAN fiber. $\mathrm{FBG}_{3}$ is also written in SMF28 fiber and has a center wavelength $\lambda_{3}=1873 \mathrm{~nm}$. All three FBGs have a peak reflectivity $R>99 \%$. In both branches, the gain fiber is double-cladding ZBLAN fiber manufactured by IRphotonics/Thorlabs, doped with 8,000 ppm $\mathrm{Tm}^{3+}$ [4]. The $\mathrm{Tm}^{3+}:$ ZBLAN fibers are coupled to the SMF-28 fibers through mechanical splices (represented by the ' $x$ ' in Fig. 1) with a loss of $\sim 2 \mathrm{~dB}$ per pair.

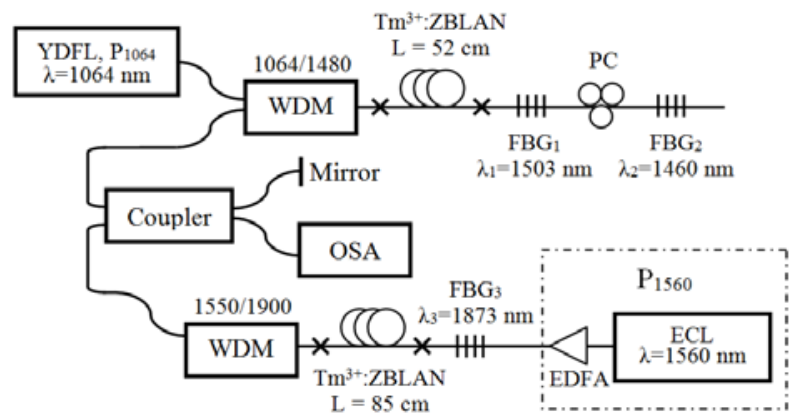

Fig. 1. $\mathrm{Tm}^{3+}$ :ZBLAN three-wavelength fiber laser configuration.

The two branches are both connected to a shared branch consisting of a coupler and a gold-tipped fiber mirror. The coupler has a 50/50 splitting ratio at $\lambda_{1}$ and $\lambda_{2}$ and an $87 / 13$ ratio at $\lambda_{3}$ (87\% to the fiber mirror). One port of the coupler is connected to the fiber mirror which serves as a reflector for both cavities, with a reflectivity above $90 \%$ at the lasing wavelengths. The other port is connected to a YOKOGAWA AQ6375 optical spectrum analyzer (OSA).

\section{RESUlTS AND DISCUSSION}

Fig. 2 shows the output power at $\lambda_{1}$ and $\lambda_{2}$ as a function of $\mathrm{P}_{1064}$, as well as the output power at $\lambda_{3}$ as a function of $\mathrm{P}_{1560}$. First, we characterize the two branches operating independently. As shown in Fig. 2(a), lasing at $\lambda_{2}$ occurs first at a threshold $\mathrm{P}_{1064}$ of $522 \mathrm{~mW}$. As $\mathrm{P}_{1064}$ is increased further, single-wavelength lasing at $\lambda_{2}$ continues to increase with 2.2\% slope efficiency. When $\mathrm{P}_{1064}$ reaches $1144 \mathrm{~mW}$, 
lasing at $\lambda_{1}$ begins and continues with $3.4 \%$ slope efficiency. As $\mathrm{P}_{1064}$ is increased to around $1580 \mathrm{~mW}$, dualwavelength lasing at $\lambda_{1}$ and $\lambda_{2}$ occurs with approximately equal output powers. As $\mathrm{P}_{1064}$ is increased further, power at $\lambda_{2}$ keeps a saturated output of $15 \mathrm{~mW}$, while power at $\lambda_{1}$ continues to increase up to a maximum output of $21 \mathrm{~mW}$ when $\mathrm{P}_{1064}$ is $1859 \mathrm{~mW}$. Fig. 2(b) shows the output power at $\lambda_{3}$ as a function of $\mathrm{P}_{1560}$. Lasing at $\lambda_{3}$ begins at a threshold of $126 \mathrm{~mW}$ and continues to increase with $\mathrm{P}_{1560}$ with $2.5 \%$ slope efficiency. The maximum output at $\lambda_{3}$ is $13 \mathrm{~mW}$ when $\mathrm{P}_{1560}$ is $637 \mathrm{~mW}$. Following this, the output powers at $\lambda_{1}, \lambda_{2}$ and $\lambda_{3}$ were re-measured with the second pump turned on. In both Fig. 2(a) and 2(b), the output curves are identical regardless of the state of the second pump, demonstrating the independence of the two branches.

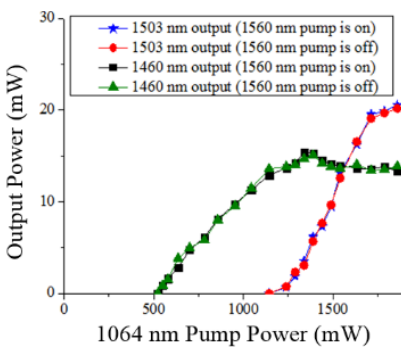

(a)

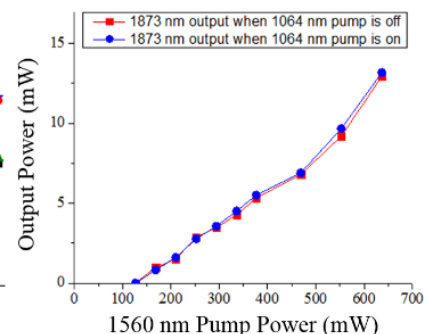

(b)
Fig. 2 Measured output power (a) at $\lambda_{1}$ and $\lambda_{2}$ as a function of $\mathrm{P}_{1064}$ when $\mathrm{P}_{1560}$ is on and off, (b) at $\lambda_{3}$ as a function of $\mathrm{P}_{1560}$ when $\mathrm{P}_{1064}$ is on and off.

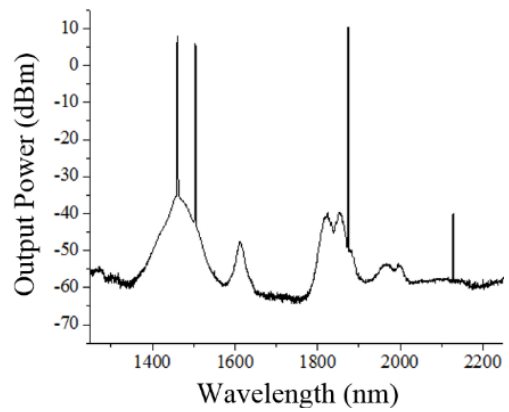

Fig. 3 Output spectrum for three-wavelength operation when $\mathrm{P}_{1064}=1520$ $\mathrm{mW}$ and $\mathrm{P}_{1560}=480 \mathrm{~mW}$.

Fig. 3 shows the output spectrum when $\mathrm{P}_{1064}$ is 1520 $\mathrm{mW}$ and $\mathrm{P}_{1560}$ is $480 \mathrm{~mW}$, which are also the pump powers used for the single-pass absorption measurements described below. Three lasing lines at $\lambda_{1}, \lambda_{2}$, and $\lambda_{3}$ are observed with peak values all above $5 \mathrm{~mW}$. Note that the peak at $2128 \mathrm{~nm}$ is an artifact of the OSA from the higher-order diffraction of $\mathrm{P}_{1064}$. The laser's output power stability is also investigated, and the peak power fluctuations of all three lasing wavelengths are found to be within $1.5 \mathrm{~dB}$ over 30 minutes.

We then use the three-wavelength laser for single-pass NIR absorption measurements on 12 samples of water in acetone, with water concentrations ranging from $0.55 \mathrm{M}$ to 11.4 M. GRIN lenses are used to couple the laser output into a $1 \mathrm{~cm}$ quartz cuvette containing the samples. The data is analyzed as in reference [5].
As shown in Fig. 4, the absorption at each lasing wavelength as a function of water concentration shows linear trends. The two blue lines represent the 99\% confidence intervals, indicating the detection limits as in reference [5]. The molar absorptivity, $\varepsilon$, can be calculated from the slope of each linear fit (red line). The lowest detection limit (about 2.8 M) is observed through the 1460 $\mathrm{nm}$ absorbance, presumably due to the larger absorption cross section and the more stable operation of the laser. The $\varepsilon$ obtained from each individual $\lambda$ is $10-20 \%$ less than theoretical value. An analysis considering solvatochromic effects at all three wavelengths is currently under way, and it appears as if the method can provide $\varepsilon$ values which are consistent with those in the literature.
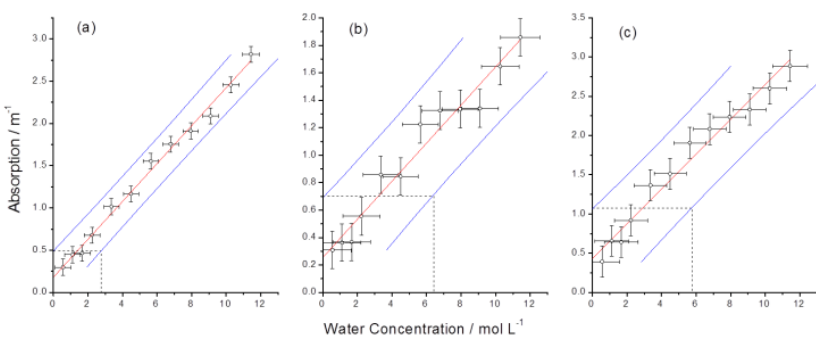

Fig. 4 Water in acetone absorbance using fiber laser at (a) $1460 \mathrm{~nm}$, (b) $1503 \mathrm{~nm}$ and (c) $1873 \mathrm{~nm}$.

\section{SUMMARY}

We have demonstrated a three-wavelength $\mathrm{Tm}^{3+}$ :ZBLAN fiber laser emitting simultaneously at 1460 $\mathrm{nm}, 1503 \mathrm{~nm}$ and $1873 \mathrm{~nm}$. In addition, we have demonstrated its application for water detection using single-pass absorption measurements. This method can be easily extended to the detection of impurities in more relevant solvents, such as water in lubricant oils or water in hydrocarbon fuel, as the laser wavelengths can be easily tailored to match the profile of the solvent.

\section{REFERENCES}

[1] P. Peterka, I. Kasik, A. Dhar, B. Dussardier, and W. Blanc "Theoretical modeling of fiber laser at $810 \mathrm{~nm}$ based on thuliumdoped silica fibers with enhanced H-3(4) level lifetime," Optics Express, vol. 19, pp. 2773-2781, Jan 2011.

[2] G. S. Qin, S. H. Huang, Y. Feng, A. Shirakawa, and K. I. Ueda, "Multiple-wavelength up-conversion laser in Tm3+-doped ZBLAN glass fiber," IEEE Photonics Technology Letters, vol. 17, pp. 18181820, Sep 2005.

[3] B. Frison, A. R. Sarmani, L. R. Chen, X. Gu, and M. Saad, "Dualwavelength S-band Tm3+: ZBLAN fibre laser with $0.6 \mathrm{~nm}$ wavelength spacing," Electronics Letters, vol. 49, pp. 60-61, Jan 2013.

[4] K. Ramaswamy, C. Jia, M. Dastmalchi, L. R. Chen, and M. Saad, "Dual-band 810/1480 nm $\mathrm{Tm}^{3+}$ :ZBLAN fiber laser," in Photonics Conference (IPC), 2013 IEEE, 2013, pp. 273-274.

[5] H.-P. Loock and P. D. Wentzell, "Detection limits of chemical sensors: Applications and misapplications," Sensors and Actuators B: Chemical, vol. 173, pp. 157-163, Oct 2012. 